\title{
SAMPLING QUANTUM NONLOCAL CORRELATIONS WITH HIGH PROBABILITY
}

\author{
C. E. GONZÁLEZ-GUILLÉN, C. H. JIMÉNEZ, C. PALAZUELOS, AND I. VILLANUEVA
}

\begin{abstract}
It is well known that quantum correlations for bipartite dichotomic measurements are those of the form $\gamma=\left(\left\langle u_{i}, v_{j}\right\rangle\right)_{i, j=1}^{n}$, where the vectors $u_{i}$ and $v_{j}$ are in the unit ball of a real Hilbert space. In this work we study the probability of the nonlocal nature of these correlations as a function of $\alpha=\frac{m}{n}$, where the previous vectors are sampled according to the Haar measure in the unit sphere of $\mathbb{R}^{m}$. In particular, we prove the existence of an $\alpha_{0}>0$ such that if $\alpha \leq \alpha_{0}, \gamma$ is nonlocal with probability tending to 1 as $n \rightarrow \infty$, while for $\alpha>2$, $\gamma$ is local with probability tending to 1 as $n \rightarrow \infty$.
\end{abstract}

\section{INTRODUCTION}

It is well known that local measurements on entangled bipartite quantum states can lead to correlations which cannot be explained by Local Hidden Variable Models (LHVM) [7]. This phenomenon, known as quantum nonlocality, is one of the most relevant features of quantum mechanics. In fact, though initially discovered in the context of foundations of quantum mechanics, during the last decade quantum nonlocality has become a crucial resource in many applications; some of them are quantum cryptography ([1, [2, [15]), communication complexity ([8]) and random number generators ([14, [16]). In this work, we will consider a particularly simple but very interesting context, where two spatially separated observers, Alice and Bob, perform dichotomic (two-outcome) measurements on a bipartite quantum state $\rho$, each on their part of the system. The simplicity of this scenario has made it the natural one to start developing the previously mentioned applications and also in the experimental verification of the quantum nonlocality phenomenon (see for instance [4], [5]).

According to the postulates of quantum mechanics, a two-outcome measurement for Alice (resp. Bob) is given by $\left\{A^{+}, A^{-}\right\}$(resp. $\left\{B^{+}, B^{-}\right\}$), where $A^{ \pm}$(resp. $B^{ \pm}$) are projectors acting on a Hilbert space and summing to the identity. We define the observable corresponding to Alice's (Bob's) measurement as $A=A^{+}-A^{-}$ $\left(B=B^{+}-B^{-}\right)$. The joint correlation of Alice's and Bob's measurement results, denoted by $a$ and $b$ respectively, is $\langle a b\rangle=\operatorname{tr}(A \otimes B \rho)$. Motivated by this, we say that $\gamma=\left(\gamma_{i, j}\right)_{i, j=1}^{n}$ is a quantum correlation matrix and denote by $\gamma \in \mathcal{Q}$, if there 
exist a density matrix $\rho$ acting on a tensor product of Hilbert spaces $H_{1} \otimes H_{2}$ and two families of contractive self-adjoint operators $\left\{A_{i}\right\}_{i=1}^{n},\left\{B_{i}\right\}_{i=1}^{n}$ acting on $H_{1}$ and $H_{2}$ respectively such that

$$
\gamma_{i, j}=\operatorname{tr}\left(A_{i} \otimes B_{j} \rho\right) \text { for every } i, j=1, \cdots, n .
$$

That is, $\gamma$ is a matrix whose entries are the correlations obtained in an AliceBob scenario where each of the observers can choose among $n$ different possible dichotomic measurements. On the other hand, we say that $\gamma=\left(\gamma_{i, j}\right)_{i, j=1}^{n}$ is a local correlation matrix if it belongs to the convex hull

$$
\mathcal{L}=\operatorname{conv}\left\{\left(\alpha_{i} \beta_{j}\right)_{i, j=1}^{n}, \alpha_{i}= \pm 1, \beta_{j}= \pm 1, i, j=1, \cdots, n\right\} .
$$

Local correlation matrices are precisely those whose entries are the correlations obtained in an Alice-Bob scenario when the measurement procedure can be explained by means of a LHVM. It is well known ([17]) that $\mathcal{L}$ and $\mathcal{Q}$ are convex sets satisfying

$$
\mathcal{L} \varsubsetneqq \mathcal{Q} \varsubsetneqq K_{G} \mathcal{L}
$$

where $1.67696 \ldots \leq K_{G} \leq 1.78221 \ldots$ is the so called Grothendieck's constant 2 . Indeed, the first strict inclusion exactly means that there exist quantum correlations which cannot be explained by means of a LHVM (what we have called quantum nonlocality above) while the second inclusion is a consequence of Grothendieck's inequality (see Theorem 1.4 below) and a result proved by Tsirelson ([17]) which states that $\gamma=\left(\gamma_{i, j}\right)_{i, j=1}^{n}$ is a quantum correlation matrix if and only if there exist a real Hilbert space $H$ and unit vectors $u_{1}, \cdots, u_{n}, v_{1}, \cdots, v_{n}$ in $H$ such that

$$
\gamma_{i, j}=\left\langle u_{i}, v_{j}\right\rangle \text { for every } i, j=1, \cdots, n \text {. }
$$

As we just mentioned, we know of the existence of quantum correlations which are nonlocal. A natural question appears now: how common is nonlocality among quantum correlations? That is, if we pick "randomly" a quantum correlation, which is the probability that it is nonlocal? To study this problem, we first need to choose a probability distribution on the set of quantum correlations, in other words, a way of sampling these matrices. We see at least two natural candidates for this. At first sight, it would seem from expression (0.1) that a natural procedure would be sampling on the set of states $\rho$ and on the set of families of self-adjoint and contractive operators $A_{1}, \cdots, A_{n}, B_{1}, \cdots, B_{n}$. The problem with this approach is twofold. First, we do not know a natural probability measure on the set of selfadjoint contractive operators. Second, it seems that we would need to allow for Hilbert spaces of very high dimension 3 .

\footnotetext{
${ }^{1}$ A density matrix is a positive operator $\rho: H \rightarrow H$ acting on a Hilbert space $H$ with $\operatorname{tr}(\rho)=1$. ${ }^{2}$ The exact value of the Grothendieck's constant is still unknown.

${ }^{3}$ It is known ([17]) that every quantum correlation $\gamma=\left(\gamma_{i, j}\right)_{i, j=1}^{n}$ can be written as in (0.1) by using a Hilbert space of dimension exponential in $n$ and, furthermore, such a dimension is required in order to describe the extreme points of $\mathcal{Q}$.
} 
So, we look for the second candidate: looking at the equivalent reformulation (0.3) of a quantum correlation, we do have a natural sampling procedure: we can sample the vectors $u_{1}, \cdots, u_{n}, v_{1}, \cdots, v_{n}$ independently uniformly distributed on the unit sphere of $\mathbb{R}^{m}$. It is well known that this is exactly the same as sampling independent normalized $m$-dimensional gaussian vectors.

Our results will depend on the relation between the dimension $m$ and the number of questions $n$. As we will show later, it is very easy to see that if one fixes any finite $m$, the probability that a quantum correlation matrix sampled according to the previous procedure is nonlocal tends to one as $n$ tends to infinity. However, this kind of sampling, though interesting to obtain quantum nonlocal correlations, does not say much about our problem, since the set of quantum correlation matrices of order $n$ which can be obtained with a fixed $m$ is very small.

We are interested in the case where $m$ and $n$ are of the same order. In that case we are sampling on a representative set of quantum correlation matrices. The main result of our work can be condensed as:

Theorem 0.1. Let $n$ and $m$ be two natural numbers and $\alpha=\frac{m}{n}$. Let us consider $2 n$ vectors $u_{1}, \cdots, u_{n}, v_{1}, \cdots, v_{n}$ sampled independently according to the Haar measure on the unit sphere of $\mathbb{R}^{m}$ and let us denote by $\gamma=\left(\left\langle u_{i}, v_{j}\right\rangle\right)_{i, j=1}^{n}$ the corresponding quantum correlation matrix.

a) If $\alpha \leq \alpha_{0} \approx 0.004$ then $\gamma$ is nonlocal with probability tending to one as $n$ tends to infinity.

b) If $\alpha>2$, then $\gamma$ is local with probability tending to one as $n$ tends to infinity.

This result shows clearly the need of studying the problem as a function of the parameter $\alpha=\frac{m}{n}$. One possible way to think of this problem is the following: say that we want to sample our vectors on a space of large dimension $m$. In that case, how many vectors $u_{1}, \cdots, u_{n}, v_{1}, \cdots, v_{n}$ will we need to sample in order to have nonlocality with high probability? Our results show that $n=\frac{m}{2}$ will be too few vectors, whereas $n=\frac{m}{\alpha_{0}}$ will be enough.

There is a considerable gap between $\alpha_{0}$ and 2. Our techniques could be refined to slightly increase the bound $\alpha_{0}$, but they will never reach the relevant case $\alpha_{0}=1$. From the other side, our proof of part b) suggests that a more clever argument could lead to replace 2 by $K_{G}$, but again our present approach does not seem to allow for further improvement. Along these lines, it is plausible that a relation between $\alpha$ and $K_{G}$ describes interesting behaviors of our correlation matrices.

It would be very interesting to understand the problem for the values $\alpha \in$ $\left(\alpha_{0}, 2\right)$ both by reducing this gap and by studying the existence, or not, of a sharp threshold behaviour of the probability of nonlocality. 
Interestingly enough, we will see below that if one samples normalized vectors whose entries are independent Bernoulli variables, the probability of obtaining a nonlocal correlation matrix is zero, since all of them will be local. This means that, in contrast to many other contexts in random matrix theory, sampling gaussian and Bernouilli random variables in our problem leads to completely different conclusions.

In order to prove Theorem 0.1 we will use a result previously proved in [10] on random matrix theory. Similar techniques were previously used in [3] in order to study the dual problem; that is, how likely it is for a random (in some sense) XOR game to have a maximum quantum value strictly bigger than a maximum classical value. In that case, the authors studied the values $\omega^{*}(A)$ and $\omega(A)$ for random matrices $A=\left(a_{i, j}\right)_{i, j=1}^{n}$, where

$$
\omega^{*}(A)=\sup \left\{\sum_{i, j=1}^{n} a_{i, j} \gamma_{i, j}: \gamma \in \mathcal{Q}\right\} \text { and } \omega(A)=\sup \left\{\sum_{i, j=1}^{n} a_{i, j} \gamma_{i, j}: \gamma \in \mathcal{L}\right\} \text {. }
$$

They concluded that, for any given $\epsilon>0, \omega^{*}(A) \geq(2-\epsilon) n^{\frac{3}{2}}$ and $\omega(A) \leq$ $1.6651 \ldots n^{\frac{3}{2}}$ with probability $1-o(1)$ as $n \rightarrow \infty$ in both cases. This result is the starting point for the proof of our Theorem 0.1. Note that stating $\frac{\omega^{*}(A)}{\omega(A)}>1$ for some $A$ 's is a reformulation (in a quantitive way) of the fact that $\mathcal{L} \varsubsetneqq \mathcal{Q}$. The elements $A$ 's are usually called correlation Bell inequalities (or XOR-games in the context of computer science) and the fact that $\frac{\omega^{*}(A)}{\omega(A)}>1$ is usually referred to as a Bell inequality violation.

The paper is organized as follows. In the first section we briefly introduce some basic results which will be used along the whole paper. The proof of Theorem 0.1 is presented in Section 2 and Section 3. The proof of part a) of the theorem, based on some results on random matrix theory, is given in Section 2, while Section 3 deals with the proof of part b).

\section{Preliminary Results}

For completeness and to simplify the reading of the paper, we state in this section the known, or essentially known, previous results which we use along the paper. The following proposition can be easily deduced from [11, Lemma 2.2].

Proposition 1.1. Let $\mathcal{G}_{n}$ be the gaussian measure on $\mathbb{R}^{n}$ and let $L \subset \mathbb{R}^{n}$ be a $k$-dimensional subspace. For a vector $g=\left(g_{1}, \cdots, g_{n}\right) \in \mathbb{R}^{n}$, let $\bar{g}=\frac{g}{\|g\|}$ and let $P_{L}(\bar{g})$ denote the orthogonal projection of $\bar{g}$ onto $L$. Then, for any $0<\rho<1$ we

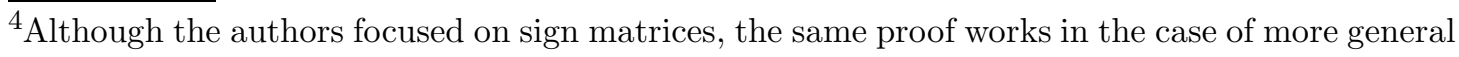
random matrices.
} 
have

$$
\mathcal{G}_{n}\left(\left(g_{1}, \cdots, g_{n}\right) \in \mathbb{R}^{n}: \quad\left\|P_{L}(\bar{g})\right\| \geq \frac{1}{1-\rho} \sqrt{\frac{k}{n}}\right) \leq e^{-\frac{\rho^{2} k}{4}}
$$

and

$$
\mathcal{G}_{n}\left(\left(g_{1}, \cdots, g_{n}\right) \in \mathbb{R}^{n}: \quad\left\|P_{L}(\bar{g})\right\| \leq(1-\rho) \sqrt{\frac{k}{n}}\right) \leq e^{-\frac{\rho^{2} k}{4}} .
$$

Remark 1.1. As we already mentioned in the Introduction, it is completely equivalent to sample a unit vector $u \in S^{n-1}$ according to the Haar measure $\mu_{n}$ to sample normalized gaussian vectors $g=\frac{1}{\left\|\left(g_{1}, \cdots, g_{n}\right)\right\|}\left(g_{1}, \cdots, g_{n}\right)$. That is, both probability distributions are exactly the same (see [6, Section 3.3] for a more complete explanation). In particular, Theorem 0.1 can be equivalently stated as it is in Theorem 2.3 and Theorem 3.1. Moreover, we can also state the previous proposition in a completely analogous form for unitary vectors and obtain:

$$
\mu_{n}\left(u \in S^{n-1}: \quad\left\|P_{L}(u)\right\| \geq \frac{1}{1-\rho} \sqrt{\frac{k}{n}}\right) \leq e^{-\frac{\rho^{2} k}{4}},
$$

and

$$
\mu_{n}\left(u \in S^{n-1}: \quad\left\|P_{L}(u)\right\| \leq(1-\rho) \sqrt{\frac{k}{n}}\right) \leq e^{-\frac{\rho^{2} k}{4}}
$$

We say that a real random $n \times n$ matrix $M$ is bi-orthogonally invariant if the distribution on $M_{n}(\mathbb{R})$ of $M$ is equal to that of $O_{1} M O_{2}$ for any orthogonal matrices $O_{1}$ and $O_{2}$. It is well known and easy to check that gaussian matrices are biorthogonally invariant.

The following result is probably well known, but we have not found a reference for it. We write a proof, following the ideas of [13, Lemma 4.3.10].

Proposition 1.2. Let $A \in M_{n}(\mathbb{R})$ be an $n \times n$ random matrix in some probability space $(\Xi, \mathbb{P})$. If $A$ is bi-orthogonally invariant then there exist random matrices $U$ and $V$ in $(\Xi, \mathbb{P})$ such that

(i) $U, V$ follow the Haar distribution in the orthogonal group $\mathcal{O}(n)$.

(ii) $U$ and $V$ are independent.

(iii) $U$ and $V$ are the matrices whose columns are respectively the left and right singular vectors associated to the ordered singular values of $A$.

Proof. For simplicity, we will assume that the set of matrices with repeated singular values has zero measure (as it happens in the gaussian case, which is the one we will use here). In this case, the singular value decomposition is unique with probability 
one up to the choice of the sign of the right (or left) singular vectors 5 . Let $A$ be a random matrix defined in some space $(\Xi, \mathbb{P})$, and let $A(\xi)=U(\xi) \Sigma(\xi) V^{*}(\xi)$ be the singular value decomposition of $A(\xi)$ where the singular values of $\Sigma(\xi)$ are ordered in decreasing order and the sign (of the first non zero coordinate) of the right singular vectors are taken at random with probability $1 / 2.6$

The random matrices $U$ and $V$ fulfill (iii) by construction. To prove (i) and (ii) it is enough to show that for any $B_{1}, B_{2} \subset \mathcal{O}(n)$ and $\Delta \subset M_{n}(\mathbb{R})$ Borel sets, we have

$$
\mathbb{P}\left(U(\xi) \in B_{1}, \Sigma(\xi) \in \Delta, V^{*}(\xi) \in B_{2}\right)=\mu_{n}\left(B_{1}\right) \mathbb{P}(\Sigma(\xi) \in \Delta) \mu_{n}\left(B_{2}\right),
$$

where $\mu_{n}$ is the Haar measure on the orthogonal group.

It follows from the biorthogonal invariance of $A$ that for any two (fixed for now) orthogonal matrices $O_{1}$ and $O_{2}$, the random matrix $A^{\prime}=O_{1} A O_{2}$ has the same distribution as $A$. Defining $U^{\prime}=O_{1} U, V^{* \prime}=V^{*} O_{2}$, it is clear that $A^{\prime}=U^{\prime} \Delta V^{* \prime}$ is a singular value decomposition of $A^{\prime}$ verifying our requirements. Therefore

$\mathbb{P}\left(U(\xi) \in B_{1}, \Sigma(\xi) \in \Delta, V^{*}(\xi) \in B_{2}\right)=\mathbb{P}\left(O_{1} U(\xi) \in B_{1}, \Sigma(\xi) \in \Delta, V^{*}(\xi) O_{2} \in B_{2}\right)$

If we now let $O_{1}$ and $O_{2}$ be distributed according to the Haar measure $\mu_{n}$ on different probability spaces $\left(\Xi^{\prime}, \mathbb{P}^{\prime}\right)$ and $\left(\Xi^{\prime \prime}, \mathbb{P}^{\prime \prime}\right)$ respectively, we get

$$
\begin{aligned}
& \mathbb{P}\left(U(\xi) \in B_{1}, \Sigma(\xi) \in \Delta, V^{*}(\xi) \in B_{2}\right) \\
& =\left(\mathbb{P}^{\prime} \otimes \mathbb{P} \otimes \mathbb{P}^{\prime \prime}\right)\left(U^{\prime}\left(\xi, \xi^{\prime}\right) \in B_{1}, \Sigma(\xi) \in \Delta, V^{\prime *}\left(\xi, \xi^{\prime \prime}\right) \in B_{2}\right) \\
& =\iiint \chi_{B_{1}}\left(O_{1}\left(\xi^{\prime}\right) U(\xi)\right) \chi_{\Delta}(\Sigma(\xi)) \chi_{B_{2}}\left(V^{*}(\xi) O_{2}\left(\xi^{\prime \prime}\right)\right) d \mathbb{P}(\xi) d \mathbb{P}^{\prime}\left(\xi^{\prime}\right) d \mathbb{P}^{\prime \prime}\left(\xi^{\prime \prime}\right) \\
& =\int\left(\int \chi_{B_{1}}\left(O_{1}\left(\xi^{\prime}\right) U(\xi)\right) d \mathbb{P}^{\prime}\left(\xi^{\prime}\right)\right) \chi_{\Delta}(\Sigma(\xi))\left(\int \chi_{B_{2}}\left(V^{*}(\xi) O_{2}\left(\xi^{\prime \prime}\right)\right) d \mathbb{P}^{\prime \prime}\left(\xi^{\prime \prime}\right)\right) d \mathbb{P}(\xi) \\
& =\int \mu_{n}\left(B_{1}\right) \chi_{\Delta}(\Sigma(\xi)) \mu_{n}\left(B_{2}\right) d \mathbb{P}(\xi) \\
& =\mu_{n}\left(B_{1}\right) \mathbb{P}(\Sigma(\xi) \in \Delta) \mu_{n}\left(B_{2}\right),
\end{aligned}
$$

where the fourth equality follows from the rotational invariance of the Haar measure.

Remark 1.2. We will use at several points of the paper the following easy consequence of Proposition [1.2, For every $n \in \mathbb{N}$ there exists a probability space $\Xi$

\footnotetext{
${ }^{5}$ The general case follows by considering the set $\mathcal{V}_{i}$ of right singular vectors associated to the singular value $s_{i}$, and taking random choices of orthonormal vectors $\mathcal{V}_{i}$ as the associated columns of the matrix $V$. The measure in $\mathcal{V}_{i}$ is the induced by the Haar measure, that is, the measure invariant under unitary transformations of $\mathcal{V}_{i}$ into itself.

${ }^{6}$ We see $A(\xi)=U(\xi) \Sigma(\xi) V^{*}(\xi)$ with $U(\xi)=U_{0}(\xi) S(\zeta)$ and $V^{*}(\xi)=S(\zeta) V_{0}^{*}(\xi)$, where $U_{0}(\xi)$ and $V_{0}(\xi)$ are a particular choice of matrices in the singular value decomposition and $S(\zeta)$ is a diagonal random matrix with even iid \pm 1 Bernoulli entries.
} 
with three $n \times n$ random matrices $A, U, V$ defined on it such that $A$ is a gaussian matrix, $U, V$ are independent and Haar distributed in $\mathcal{O}(n)$, and for almost every $\xi \in \Xi, U(\xi)$ and $V(\xi)$ are the right and left singular values of $A(\xi)$ arranged in decreasing order of the singular values.

We will need the Marcenko-Pastur law, describing the distribution of the singular values of random matrices:

Theorem 1.3 (Marcenko-Pastur law, [12]). Let $A$ be an $n \times n$ random matrix whose entries $a_{i j}$ are independent real random variables with mean 0 and variance 1. Let $C \in[0,2]$. With probability $1-o(1)$, the number of singular values $\lambda$ of $A$ that satisfy $\lambda \geq C \sqrt{n}$ is $(f(C)-o(1)) n$ where

$$
f(C)=\frac{1}{2 \pi} \int_{x=C^{2}}^{4} \sqrt{\frac{4}{x}-1} d x .
$$

Here, we say that $h=h(n)$ is o(1) if and only if $\lim _{n \rightarrow \infty} h(n)=0$.

Finally, we state for completeness the version of Grothendieck's inequality most useful for our purposes (see [9, Page 172]).

Theorem 1.4 (Grothendieck's inequality). There exists a universal constant $K_{G}$, such that for every natural number $n$ and for every real matrix $\left(a_{i, j}\right)_{i, j=1}^{n}$ we have

$$
\sup \left\{\left|\sum_{i, j=1}^{n} a_{i, j}\left\langle x_{i}, y_{j}\right\rangle\right|: x_{i}, y_{j} \in B_{H}\right\} \leq K_{G} \sup \left\{\left|\sum_{i, j=1}^{n} a_{i, j} s_{i} t_{j}\right|: s_{i}, t_{j}= \pm 1\right\},
$$

where the first supremum runs over elements $x_{1}, \cdots, x_{n}, y_{1}, \cdots, y_{n}$ in the unit ball of a real Hilbert space $H$.

The exact value of $K_{G}$ is still unknown but we have $1.67696 \ldots \leq K_{G} \leq 1.78221 \ldots$.

\section{A LOWER Bound For $\alpha_{0}$ : PART A) of Theorem 0.1}

The following result is implicit in the paper [3]. It provides an abundance of quantum nonclassical correlations and it is the starting point of our work.

Proposition 2.1. Let $U=\left(u_{i, j}\right)_{i, j=1}^{n}, V=\left(v_{i, j}\right)_{i, j=1}^{n}$ be two independent orthogonal random matrices distributed according to the Haar measure on the orthogonal group $\mathcal{O}(n)$. Let $\alpha \in(0,1)$ and $m=\alpha n$. We also denote $\delta=f^{-1}(\alpha)$, where $f$ is the Marcenko-Pastur densitiy function as in Theorem 1.3. Let $\gamma_{i, j}=\left\langle\frac{\sqrt{n}}{\sqrt{m}} u_{i}, \frac{\sqrt{n}}{\sqrt{m}} v_{j}\right\rangle$ with $u_{i}=\left(u_{i, k}\right)_{k=1}^{m} v_{j}=\left(v_{j, k}\right)_{k=1}^{m}$. Then there exists an $n \times n$ matrix $A=\left(a_{i, j}\right)_{i, j=1}^{n}$ such that, with probability $1-o(1)$,

$$
\sum_{i, j=1}^{n} a_{i, j} \gamma_{i, j} \geq(\delta-o(1)) n^{\frac{3}{2}} \text { and } \omega(A) \leq 1.6651 \ldots n^{\frac{3}{2}}
$$


Proof. We consider $A, U, V$ distributed as in Remark 1.2. So, $A=U \Sigma V^{*}$, with $\Sigma$ is the diagonal matrix of the singular values which we may asume arranged in decreasing order. Let $\lambda_{1}, \ldots, \lambda_{m}$ be the greatest $m$ singular values of $A$. According to our choice of $\delta$, it follows from Theorem 1.3 that $\lambda_{m} \geq(\delta-o(1)) \sqrt{n}$ with probability $1-o(1)$. Then, we have

$$
\sum_{i, j=1}^{n} a_{i, j}\left\langle\frac{\sqrt{n}}{\sqrt{m}} u_{i}, \frac{\sqrt{n}}{\sqrt{m}} v_{j}\right\rangle=\frac{n}{m} \sum_{i, j=1}^{n} a_{i, j}\left\langle u_{i}, v_{j}\right\rangle=\frac{n}{m} \sum_{k=1}^{m} \lambda_{k} \geq(\delta-o(1)) n^{\frac{3}{2}} .
$$

This proves the first inequality of our statement. For the second one, note that $A$ is a gaussian matrix. Then the result follows exactly as in [3, Theorem 5] from the Chernoff bound:

$$
\operatorname{Pr}\left(\left|\sum_{k=1}^{n} a_{k} X_{k}\right| \geq t\right) \leq 2 e^{-\frac{t^{2}}{2\|a\|^{2}}}
$$

where $X_{k}$ are normalized real gaussian variables and $a=\left(a_{1}, \ldots, a_{n}\right) \in \mathbb{R}^{n}$, taking $t=\left(2 \sqrt{\ln 2}+2 \frac{\sqrt{\ln n}}{\sqrt{n}}\right) n^{3 / 2}$ and applying a union bound argument.

Now we can state and prove the first part of Theorem 0.1. It states that most correlations will be nonlocal when $m$ is of the order $\alpha_{0} n$. The idea of the proof is the following: Proposition 2.1 shows that for that order of $m$ the first $m$ rows/columns of two Haar distributed orthogonal matrices generate a nonlocal quantum correlation. It also provides a gaussian matrix $A$ that certifies this nonlocality. On the other hand, the following theorem shows that the first $m$ columns of a gaussian matrix are "close", in an appropriate sup-euclidean norm, to the first $m$ columns of a Haar distributed orthogonal matrix.

Theorem 2.2. [10, Theorem 1.1] Let $n$ and $m$ be two natural numbers such that $\alpha=\frac{m}{n} \in(0,1)$. Then, there exist matrices $Y_{n}=\left(y_{i, j}\right)_{i, j=1}^{n}$ and $U_{n}=\left(u_{i j}\right)_{i, j=1}^{n}$ whose $2 n^{2}$ entries are real random variables defined on the same probability space $\Xi$ such that

(i) $\left\{y_{i, j} ; 1 \leq i, j \leq n\right\}$ are independent normalized random gaussian variables,

(ii) $U_{n}$ is an orthogonal matrix distributed according to the Haar measure,

(iii) If we set $F_{i}^{m}\left(Y_{n}-\sqrt{n} U_{n}\right)$ the $i$-th row of the matrix $Y_{n}-\sqrt{n} U_{n}$ truncated to its first $m$ entries, we have

$$
\mathbb{P}_{\Xi}\left(\sup _{i=1, \cdots, n}\left\|F_{i}^{m}\left(Y_{n}-\sqrt{n} U_{n}\right)\right\|>(1+\epsilon) \theta(\alpha) \sqrt{m}\right) \leq K n e^{C(\epsilon, \alpha) n},
$$

where here $K$ is a universal positive constant, $C(\epsilon, \alpha)>0$ is a constant depending only on $\epsilon$ and $\alpha$ and

$$
\theta(\alpha)=\sqrt{2-\frac{4}{3} \frac{\left(1-(1-\alpha)^{3 / 2}\right)}{\alpha}} .
$$


Finally, Grothendieck's inequality allows us to translate this sup-euclidean closeness into a big value of the correlation $\gamma$ when tested against the witness $A$.

Theorem 2.3. Let $G=\left(g_{i, j}\right)_{i, j=1}^{n, m}$ and $H=\left(h_{i, j}\right)_{i, j=1}^{n, m}$ be two random matrices whose entries are independent real normalized gaussian variables satisfying $\alpha=$ $\frac{m}{n} \in(0,1)$. For every $i, j=1, \cdots, n$, let $g_{i}=\left(g_{i, k}\right)_{k=1}^{m}$ and $h_{j}=\left(h_{j, k}\right)_{k=1}^{m}$ be the row vectors of $G$ and $H$ respectively. Let us denote $\bar{g}_{i}=\frac{g_{i}}{\left\|g_{i}\right\|}$ and $\bar{h}_{j}=\frac{h_{j}}{\left\|h_{j}\right\|}$. Then, if $\alpha \leq \alpha_{0} \approx 0.004$, the quantum correlation matrix given by $\gamma=\left(\left\langle\bar{g}_{i} \mid \bar{h}_{j}\right\rangle\right)_{i, j=1}^{n}$ is not local with probability $1-o(1)$.

As we will explain below, it suffices to show the result for $\alpha=\alpha_{0}$.

Proof. Following Remark 1.2 and Theorem 2.2 we may consider $A$ a gaussian matrix, $U$ and $V$ the matrices formed by the left and right singular vectors of $A$, arranged in decreasing order, and $G^{\prime}$ and $H^{\prime}$ the independent gaussian matrices whose Gram-Schmidt orthonormalization yields $U$ and $V$ respectively. Now, $G$ and $H$ from the statement are the first m columns of matrices $G^{\prime}$ and $H \sqrt{7}$. Let us denote by $\mathcal{G}_{2 n^{2}}$ the gaussian measure of dimension $2 n^{2}$. We define $u_{i}=\left(u_{i, k}\right)_{k=1}^{m}$ and $v_{j}=\left(v_{j, k}\right)_{k=1}^{m}$.

According to Proposition 2.1 we have

$$
\omega(A) \leq 1.6651 \ldots n^{\frac{3}{2}}
$$

with probability $1-o(1)$. We need to see now that $\sum_{i, j=1}^{n} a_{i, j} \gamma_{i, j}$ is greater than this value. We write

$$
\begin{gathered}
\bar{g}_{i}=\frac{\sqrt{n} u_{i}}{\sqrt{m}}+\frac{g_{i}}{\sqrt{m}}-\frac{\sqrt{n} u_{i}}{\sqrt{m}}+\bar{g}_{i}-\frac{g_{i}}{\sqrt{m}}:=\frac{\sqrt{n} u_{i}}{\sqrt{m}}+\varepsilon_{i}, \\
\bar{h}_{j}=\frac{\sqrt{n} v_{j}}{\sqrt{m}}+\frac{h_{j}}{\sqrt{m}}-\frac{\sqrt{n} v_{j}}{\sqrt{m}}+\bar{h}_{j}-\frac{h_{j}}{\sqrt{m}}:=\frac{\sqrt{n} v_{j}}{\sqrt{m}}+\sigma_{j} .
\end{gathered}
$$

Therefore, $\left|\sum_{i, j=1}^{n} a_{i, j} \gamma_{i, j}\right|=\left|\sum_{i, j=1}^{n} a_{i, j}\left\langle\bar{g}_{i}, \bar{h}_{j}\right\rangle\right|$ is lower bounded by

$$
\begin{array}{r}
\left|\sum_{i, j=1}^{n} a_{i, j}\left\langle\frac{\sqrt{n} u_{i}}{\sqrt{m}}, \frac{\sqrt{n} v_{j}}{\sqrt{m}}\right\rangle\right|-\left|\sum_{i, j=1}^{n} a_{i, j}\left\langle\frac{\sqrt{n} u_{i}}{\sqrt{m}}, \sigma_{j}\right\rangle\right| \\
-\left|\sum_{i, j=1}^{n} a_{i, j}\left\langle\varepsilon_{i}, \frac{\sqrt{n} v_{j}}{\sqrt{m}}\right\rangle\right|-\left|\sum_{i, j=1}^{n} a_{i, j}\left\langle\varepsilon_{i}, \sigma_{j}\right\rangle\right| .
\end{array}
$$

\footnotetext{
${ }^{7}$ Note that the last $n-m$ columns of $G^{\prime}$ and $H^{\prime}$ will not play any role in the proof. They are only introduced in order to apply Theorem 2.2 in a simple way.
} 
Proposition 2.1 tells us that

$$
\sum_{i, j=1}^{n} a_{i, j}\left\langle\frac{\sqrt{n} u_{i}}{\sqrt{m}}, \frac{\sqrt{n} v_{j}}{\sqrt{m}}\right\rangle \geq(\delta-o(1)) n^{\frac{3}{2}},
$$

where $\delta=f^{-1}(\alpha)$ as in Proposition 2.1.

We need now to upper bound the other three summands in (2.2). We will do this by means of Theorem 1.4. First, we need to bound the norm of the vectors $\varepsilon_{i}, \sigma_{j}$ : We do this for the $\varepsilon_{i}$ 's, since the $\sigma_{j}$ 's are totally analogous.

We note that

$$
\left\|\varepsilon_{i}\right\| \leq\left\|\frac{g_{i}}{\sqrt{m}}-\frac{\sqrt{n} u_{i}}{\sqrt{m}}\right\|+\left\|\bar{g}_{i}-\frac{g_{i}}{\sqrt{m}}\right\| .
$$

In order to bound $\left\|\bar{g}_{i}-\frac{g_{i}}{\sqrt{m}}\right\|=\left|1-\frac{\left\|g_{i}\right\|}{\sqrt{m}}\right|$, one can use very well known estimates to state that

$$
\mathcal{G}_{2 n^{2}}\left(\sup _{i=1, \cdots, n}\left\|\bar{g}_{i}-\frac{g_{i}}{\sqrt{m}}\right\|>\epsilon\right) \leq 2 n e^{-\frac{\epsilon^{2} m}{4}}
$$

for a small enough $\epsilon \in(0,1)$. Indeed, this is immediate from the corresponding bound for a fixed $i$ (see for instance [6, Corollary 2.3]) and a union bound argument. On the other hand, according to Theorem 2.2 we have

$$
\mathcal{G}_{2 n^{2}}\left(\sup _{i=1, \cdots, n}\left\|\frac{g_{i}}{\sqrt{m}}-\frac{\sqrt{n} u_{i}}{\sqrt{m}}\right\|>(1+\epsilon) \theta(\alpha)\right) \leq K n e^{C(\epsilon, \alpha) n}
$$

Thus, for a given $\epsilon>0$ we have that

$$
\mathcal{G}_{2 n^{2}}\left(\sup _{i=1, \cdots, n}\left\|\epsilon_{i}\right\|>\epsilon+\theta(\alpha)\right) \leq K^{\prime} n e^{C^{\prime}(\epsilon, \alpha) n} .
$$

On the other hand, according to Proposition 1.1 and Remark 1.1 we have

$$
\mathcal{G}_{2 n^{2}}\left(\sup _{j=1, \cdots, n} \frac{\sqrt{n}}{\sqrt{m}}\left\|u_{j}\right\|>\frac{1}{1-\epsilon}\right) \leq n e^{-\frac{\epsilon^{2} m}{4}} .
$$

We have used here that each of the $u_{j}$ is the projection onto the first $m$-coordinates of a unit vector distributed according to the Haar measure in $\mathbb{R}^{n}$, and we have also used a union bound argument to consider the supremum on $j$. Then, we can invoke Theorem 1.4 to state that for a fixed $\epsilon>0$ we have

$$
\left|\sum_{i, j=1}^{n} a_{i, j}\left\langle\varepsilon_{i}, \frac{\sqrt{n} v_{j}}{\sqrt{m}}\right\rangle\right| \leq(\epsilon+\theta(\alpha)) \frac{1}{1-\epsilon} K_{G} \omega(A)
$$

with probability larger than $1-K^{\prime \prime} n e^{C^{\prime}(\epsilon, \alpha) n}$. By following completely analogous arguments for the rest of terms in $(2.2)$ we finally get that

$$
\left|\sum_{i, j=1}^{n} a_{i, j} \gamma_{i, j}\right| \geq(\delta-o(1)) n^{\frac{3}{2}}-\left(2(\epsilon+\theta(\alpha)) \frac{1}{1-\epsilon}+(\epsilon+\theta(\alpha))^{2}\right) K_{G} \omega(A)
$$


with probability larger than $1-K_{1} n e^{-K_{2}(\epsilon, \alpha) m}$, where $K_{1}$ is a universal positive constant and $K_{2}(\epsilon, \alpha)$ is a positive constant depending only on $\epsilon$ and $\alpha$. Then, in order to have a Bell violation and using that $\epsilon$ can be made arbitrarily small, it suffices to impose that

$$
(\delta-o(1)) n^{\frac{3}{2}}>\omega(A)+\left(2 \theta(\alpha)+\theta(\alpha)^{2}\right) K_{G} \omega(A) .
$$

It follows from (2.1), the relation between $\alpha$ and $\delta$ described in Proposition 2.1 and Theorem 2.2 that for $\alpha_{0}=0.00404$ the previous inequality is verified.

It is very easy to show that for any fixed finite $m$, the probability that a quantum correlation matrix sampled according to our procedure is nonlocal tends to one as $n$ tends to infinity. We write the proof for the case $m=2$, but the reasoning extends trivially to finite $m$.

It is well known that in the case $n=2$ the element $A=\left(\begin{array}{cc}1 & 1 \\ 1 & -1\end{array}\right)$ verifies $\omega(A)=2$. On the other hand, if we define $u_{1}=(1,0), u_{2}=(0,1), v_{1}=\frac{1}{\sqrt{2}}(1,1)$, $v_{2}=\frac{1}{\sqrt{2}}(1,-1)$ we have

$$
\sum_{i, j=1}^{2} a_{i, j}\left\langle u_{i}, v_{j}\right\rangle=2 \sqrt{2} .
$$

$A$ is usually called CHSH-inequality. In particular, the quantum correlation matrix given by $\gamma=\left(\left\langle u_{i}, v_{j}\right\rangle\right)_{i, j=1}^{2}$ is nonlocal. Since the function

$$
f\left(u_{1}, u_{2}, v_{1}, v_{2}\right)=\sum_{i, j=1}^{2} a_{i, j}\left\langle u_{i}, v_{j}\right\rangle
$$

is continuous on the cartesian product of unit spheres in $\mathbb{R}^{n}, S:=S^{1} \times S^{1} \times S^{1} \times S^{1}$, we can easily conclude the existence of an open subset $B$ of $S$ such that $f_{\mid B}>2$. By considering a subset of $B$, we can assume that this set has positive Haar measure. Hence, the probability that a quantum correlation matrix sampled according to our procedure with $m=n=2$ is nonlocal is strictly larger than zero. Therefore, if we consider the same sampling as above with $n$ large, we can consider independent $2 \times 2$-blocks of $\gamma=\left(\left\langle u_{i}, v_{j}\right\rangle\right)_{i, j=1}^{n}$ and check the probability that at least one of these blocks is non local. This probability will tend to one as $n$ tends to infinity.

With the same ideas one can prove that Theorem 2.3 remains true if we let $m<\alpha_{0} n$. In particular we can also cover the case where $m$ grows sublinearly with $n$. Call $m_{n}$ to the dimension we will consider in the case $n$. If $m_{n}$ stays bounded as $n$ grows to infinity, the reasonings from the previous paragraph above apply. Otherwise, for every $n$ we consider only matrices/Bell inequalities $A$ which involve the first $\frac{m_{n}}{\alpha_{0}}$ vectors, and apply Theorem 2.3 . 
To finish this section we will show that sampling normalized vectors $u_{i}$ and $v_{j}$ whose entries are independent Bernoulli variables leads to local correlations with probability one. Indeed, if we consider such vectors $u_{i}=\frac{1}{\sqrt{m}}\left(\epsilon_{1}^{i}, \cdots, \epsilon_{m}^{i}\right)$, $v_{j}=\frac{1}{\sqrt{m}}\left(\delta_{1}^{j}, \cdots, \delta_{m}^{j}\right)$, we obtain that

$$
\left(\gamma_{i, j}\right)_{i, j=1}^{n}=\left(\frac{1}{m} \sum_{k=1}^{m} \epsilon_{k}^{i} \delta_{k}^{j}\right)_{i, j=1}^{n} .
$$

However, for a fixed $k$, we have that $\left(\gamma_{i, j}^{k}\right)_{i, j=1}^{n}=\left(\epsilon_{k}^{i} \delta_{k}^{j}\right)_{i, j=1}^{n}$ is a deterministic (so local) correlation. Since $\left(\gamma_{i, j}\right)_{i, j=1}^{n}$ is written as a convex combination of these objects, we immediately conclude that $\left(\gamma_{i, j}\right)_{i, j=1}^{n}$ is a local correlation.

\section{An upper bound For $\alpha_{0}$ : Part B) of Theorem 0.1}

Theorem 3.1. Let $G=\left(g_{i, j}\right)_{i, j=1}^{n, m}$ and $H=\left(h_{i, j}\right)_{i, j=1}^{n, m}$ be two random matrices whose entries are independent real normalized gaussian variables and let $\alpha=\frac{m}{n}$. For every $i, j=1, \cdots, n$, let $g_{i}=\left(g_{i, k}\right)_{k=1}^{m}$ and $h_{j}=\left(h_{j, k}\right)_{k=1}^{m}$ be the row vectors of $G$ and $H$ respectively. Let us denote $\bar{g}_{i}=\frac{g_{i}}{\left\|g_{i}\right\|}$ and $\bar{h}_{j}=\frac{h_{j}}{\left\|h_{j}\right\|}$. Then, if $\alpha>2$, the quantum correlation matrix given by $\gamma=\left(\left\langle\bar{g}_{i} \mid \bar{h}_{j}\right\rangle\right)_{i, j=1}^{n}$ is local with probability larger than $1-2 n e^{C(\alpha) n}$. Here, $C(\alpha) \in(0,1)$ is a constant depending only on $\alpha$.

For the proof of Theorem 3.1 we will use some elements from Banach space theory: Given an $n \times n$ matrix with real entries $\Gamma=\left(\gamma_{i, j}\right)_{i, j=1}^{n}$, we can regard this matrix as a tensor $\Gamma=\sum_{i, j=1}^{n} \gamma_{i, j} e_{i} \otimes e_{j} \in \mathbb{R}^{n} \otimes \mathbb{R}^{n}$. It will be convenient for us to introduce two tensor norms. We define

$$
\|\Gamma\|_{\ell_{\infty}^{n} \otimes_{\pi} \ell_{\infty}^{n}}=\inf \left\{\sum_{k=1}^{N}\left\|x_{i}\right\|_{\infty}\left\|y_{i}\right\|_{\infty}: \Gamma=\sum_{i=1}^{N} x_{i} \otimes y_{i}\right\},
$$

where in this definition, given a vector $z \in \mathbb{R}^{n}$, we denote $\|z\|_{\infty}=\max _{i=1, \cdots, n}\left|z_{i}\right|$. This norm is the projective tensor norm on $\ell_{\infty}^{n} \otimes \ell_{\infty}^{n}$ and it can be equivalently defined (see [9, Chapter 3]) as

$$
\|\Gamma\|_{\ell_{\infty}^{n} \otimes_{\pi} \ell_{\infty}^{n}}=\inf \left\{\sum_{k=1}^{N} \lambda_{k}: \lambda_{k} \geq 0, \Gamma=\sum_{k=1}^{N} \lambda_{k} \eta_{k}\right\},
$$

where here $\eta_{k}$ denotes the matrix associated to a determinist (so local) correlation. That is, for every $k$ we have that $\eta_{k}=a_{k} \otimes b_{k}$ for certain sign vectors $a_{k}, b_{k} \in \mathbb{R}^{n}$.

Remark 3.1. Hence, it is clear why we are interested in this norm: For a given matrix $A$, we trivially have that

$\|\Gamma\|_{\ell_{\infty}^{n} \otimes_{\pi} \ell_{\infty}^{n}} \leq 1$ if and only if $\Gamma$ is local (as a correlation matrix). 
On the other hand, we define

$$
\|\Gamma\|_{\ell_{\infty}^{n}\left(\ell_{2}^{n}\right)}=\max _{i=1, \cdots, n}\left\|\left(\gamma_{i, j}\right)_{j=1}^{n}\right\| .
$$

The following result is the key point in our proof of Theorem 3.1 ,

Theorem 3.2. Given an $n \times n$ matrix with real entries $\Gamma=\left(\gamma_{i, j}\right)_{i, j=1}^{n}$, we have that

$$
\|\Gamma\|_{\ell_{\infty}^{n} \otimes_{\pi} \ell_{\infty}^{n}} \leq \sqrt{2}\|\Gamma\|_{\ell_{\infty}^{n}\left(\ell_{2}^{n}\right)}
$$

Proof. The statement of the theorem is a reformulation of the well known fact that $\pi_{1}\left(i d: \ell_{1}^{n} \rightarrow \ell_{2}^{n}\right) \leq \sqrt{2}$, where here $\pi_{1}$ denotes the 1 -summing norm (see for instance [9, Ex 11.5]). In fact, this estimate can be easily deduced from Khintchine inequality. It means by definition that

$$
\left\|i d \otimes i d: \ell_{1}^{n} \otimes_{\epsilon} \ell_{1}^{n} \rightarrow \ell_{1}^{n}\left(\ell_{2}^{n}\right)\right\| \leq \sqrt{2} .
$$

Therefore, the statement follows by considering the adjoint map, which goes from $\ell_{\infty}^{n}\left(\ell_{2}^{n}\right)=\left(\ell_{1}^{n}\left(\ell_{2}^{n}\right)\right)^{*}$ to $\ell_{\infty}^{n} \otimes_{\pi} \ell_{\infty}^{n}=\left(\ell_{1}^{n} \otimes_{\epsilon} \ell_{1}^{n}\right)^{*}$. Here, $\epsilon$ denotes the injective tensor norm, which can be defined as the dual of the projective one.

Proof of Theorem 3.1. Let us first consider the matrix $\hat{\gamma}$ which is defined as the matrix $\gamma$ but considering the vectors $\hat{h}_{j}=\frac{1}{\sqrt{m}} h_{j}$ instead of the normalized ones $\bar{h}_{j}=\frac{1}{\left\|h_{j}\right\|} h_{j}$. Then, if we denote $\hat{\gamma}_{i}=\left(\left\langle\bar{g}_{i}, \hat{h}_{j}\right\rangle\right)_{j=1}^{n}$ for every $i=1, \cdots, n$, we have

$$
\left\|\hat{\gamma}_{i}\right\|=\frac{1}{\sqrt{m}}\left(\sum_{j=1}^{n}\left\langle\bar{g}_{i}, h_{j}\right\rangle^{2}\right)^{\frac{1}{2}} .
$$

Since $\bar{g}_{i}$ is a unit vector which is independent of the gaussian vectors $h_{j}$, we easily deduce that

$$
\mathcal{G}_{2 n^{2}}\left(\left\|\hat{\gamma}_{i}\right\| \geq \frac{1}{\sqrt{1-\epsilon}} \sqrt{\frac{n}{m}}\right)=\mathcal{G}_{n}\left(\frac{1}{\sqrt{m}}\left(\sum_{j=1}^{n} y_{j}^{2}\right)^{\frac{1}{2}} \geq \frac{1}{\sqrt{1-\epsilon}} \sqrt{\frac{n}{m}}\right) \leq e^{-\frac{n \epsilon^{2}}{4}}
$$

where $\mathcal{G}_{n}$ is the probability when we sample a vector $\left(y_{1}, \cdots, y_{n}\right)$ of independent normalized real gaussian variables and the last inequality follows from well known estimates on the norm of gaussian vectors (see for example [6, Corollary 2.3]). Therefore, if $\frac{m}{n}=\alpha>2$ we can find an $\epsilon_{0}=\epsilon(\alpha) \in(0,1)$ so that

$$
\mathcal{G}_{2 n^{2}}\left(\left\|\hat{\gamma}_{i}\right\| \geq\left(1-\epsilon_{0}\right) \frac{1}{\sqrt{2}}\right) \leq e^{-n C(\alpha)}
$$

where $C(\alpha)=\frac{\epsilon_{0}^{2}}{4}$. Moreover, by a union bound argument we obtain

$$
\mathcal{G}_{2 n^{2}}\left(\|\hat{\gamma}\|_{\ell_{\infty}^{n}\left(\ell_{2}^{n}\right)} \geq\left(1-\epsilon_{0}\right) \frac{1}{\sqrt{2}}\right) \leq n e^{-n C(\alpha)} .
$$


According to Theorem 3.2 , this shows that

$$
\mathcal{G}_{2 n^{2}}\left(\|\hat{\gamma}\|_{\ell_{\infty}^{n} \otimes_{\pi} \ell_{\infty}^{n}} \geq\left(1-\epsilon_{0}\right)\right) \leq n e^{-n C(\alpha)} .
$$

Furthermore, Remark 3.1 tells us that the previous equation is equivalent to

$$
\mathcal{G}_{2 n^{2}}\left(\sup \left\{\left|\sum_{i, j=1}^{n} a_{i, j} \hat{\gamma}_{i, j}\right|: \omega(A) \leq 1\right\} \geq 1-\epsilon_{0}\right) \leq n e^{-n C(\alpha)} .
$$

Now, according to Theorem 1.4, for every matrix with $\omega(A) \leq 1$ we have

$$
\begin{aligned}
\left|\sum_{i, j=1}^{n} a_{i, j} \gamma_{i, j}\right| & =\left|\sum_{i, j=1}^{n} a_{i, j} \hat{\gamma}_{i, j}+\sum_{i, j=1}^{n} a_{i, j}\left\langle\bar{g}_{i}, \bar{h}_{j}-\hat{h}_{j}\right\rangle\right| \\
& \leq\left|\sum_{i, j=1}^{n} a_{i, j} \hat{\gamma}_{i, j}\right|+\left|\sum_{i, j=1}^{n} a_{i, j}\left\langle\bar{g}_{i}, \bar{h}_{j}-\hat{h}_{j}\right\rangle\right| \\
& \leq\left|\sum_{i, j=1}^{n} a_{i, j} \hat{\gamma}_{i, j}\right|+K_{G} \max _{j=1, \cdots, n}\left\|\bar{h}_{j}-\hat{h}_{j}\right\| .
\end{aligned}
$$

On the other hand, we have already explained in the proof of Theorem 2.3 that for a small enough $\delta \in(0,1)$

$$
\mathcal{G}_{2 n^{2}}\left(\sup _{j=1, \cdots, n}\left\|\bar{h}_{j}-\hat{h}_{j}\right\|>\delta\right) \leq 2 n e^{-\frac{\delta^{2} m}{4}}
$$

Now, by considering a small enough $\delta=\delta(\alpha) \in(0,1)$ we conclude that

$$
\mathcal{G}_{2 n^{2}}\left(\sup \left\{\left|\sum_{i, j=1}^{n} M_{i, j} \gamma_{i, j}\right|: \omega(M) \leq 1\right\} \geq 1\right) \leq 3 n e^{-n \tilde{C}(\alpha)}
$$

where $\tilde{C}(\alpha) \in(0,1)$ is a constant depending only on $\alpha$. This finishes our proof.

\section{ACKNOWLEDGMents}

We would like to thank Jop Briet and Yeong-Cherng Liang for helpful discussions on previous versions. Author's research was supported by Spanish projects MTM2011-26912, MTM2012-30748, QUITEMAD+ and "Ramón y Cajal" program and Mexico's National Council for Science and Technology postdoctoral grant 180486.

\section{REFERENCES}

[1] A. Acin, N. Brunner, N. Gisin, S. Massar, S. Pironio, and V. Scarani, Device-independent security of quantum cryptography against collective attacks, Phys. Rev. Lett. 98, 230501 (2007).

[2] A. Acín, N. Gisin, and L. Masanes, From Bell's Theorem to Secure Quantum Key Distribution, Phys. Rev. Lett. 97, 120405 (2006). 
[3] A. Ambainis, A. Backurs, K. Balodis, D. Kravcenko, R. Ozols, J. Smotrovs, M. Virza, Quantum strategies are better than classical in almost any XOR game, Automata, Languages, and Programming Lecture Notes in Computer Science Volume 7391, 2012, 25-37.

[4] A. Aspect, Bell's inequality test: more ideal than ever, Nature 398, 189-190 (1999).

[5] A. Aspect, P. Grangier and G. Roger, Experimental Tests of Realistic Local Theories via Bell's Theorem, Phys. Rev. Lett. 47, 460 (1981).

[6] A. Barvinok, Measure Concentration. Math 710 Lecture Notes, Department of Mathematics, University of Michigan (2005).

[7] J. S. Bell, On the einstein-podolsky-rosen paradox, Physics 1, 195-200 (1964).

[8] H. Buhrman, R. Cleve, S. Massar, and R. de Wolf, Nonlocality and communication complexity, Rev. Mod. Phys. 82, 665 (2010).

[9] A. Defant and K. Floret, Tensor Norms and Operator Ideals, North-Holland, (1993).

[10] C. González-Guillén, C. Palazuelos, I. Villanueva, Euclidean distance between Haar orthogonal and gaussian matrices. arXiv:1412.3743

[11] S. Dasgupta, A. Gupta, An elementary proof of a theorem of Johnson and Lindenstrauss, Random Struct. Alg. 22(1), 60-65 (2003).

[12] V. A. Marcenko, L. A. Pastur, Distribution of eigenvalues for some sets of random matrices, Math. USSR Sbornik, 1:457-483, (1967).

[13] F. Hiai and D. Petz, The semicircle law, free random variables and entropy, American Mathematical Society Providence, (2000).

[14] S. Pironio, A. Acín, S. Massar, A. Boyer de la Giroday, D. N. Matsukevich, P. Maunz, S. Olmschenk, D. Hayes, L. Luo, T. A. Manning and C. Monroe, Random numbers certified by Bell's theorem, Nature (London) 464, 1021 (2010).

[15] U. Vazirani, T. Vidick, Fully device-independent quantum key distribution, available in arXiv:1210.1810 (2012).

[16] U. Vazirani, T. Vidick, Certifiable quantum dice - Or, exponential randomness expansion, Phil. Trans. R. Soc. A, 370, 3432-3448 (2012).

[17] B. S. Tsirelson, Some results and problems on quantum Bell-type inequalities, Hadronic J. Supp. 8(4), 329-345 (1993).

Departamento de Matemáticas del Área Industrial, E.T.S.I. Industriales, UPM, 28006 Madrid, Spain; IMI, UCM, 28040, Madrid, Spain

E-mail address: carlos.gguillen@upm.es

Departamento de Matemática, Universidad Federal de Minas Gerais

E-mail address: carloshugo@us.es

Departamento de Análisis Matemático, UCM, 28040, Madrid, Spain;, Instituto de Ciencias Matemáticas (ICMAT), 28049, Madrid. Spain

E-mail address: carlospalazuelos@ucm.es

Departamento de Análisis Matemático and IMi, UCM, 28040, Madrid, Spain

E-mail address: ignaciov@ucm.es 